\title{
Guest Editorial: Healthcare Intelligent Multi Agent Systems
}

\author{
Neil Vaughan ${ }^{1} \cdot$ Eloisa Vargiu ${ }^{2} \cdot$ Stefano Mariani $^{3} \cdot$ Sara Montagna $^{4} \cdot$ Michael I. Schumacher $^{5}$ \\ Published online: 9 July 2020 \\ (C) Springer Science+Business Media, LLC, part of Springer Nature 2020
}

This brief introductory note by the organising chairs of Healthcare Intelligent Multi-Agent Systems (HIMAS2020) provides a background to this topical collection, which accompanies the HIMAS2020 workshop. Our HIMAS2020 workshop was competitively selected in peer review to form an official part of the International Conference on Autonomous Agents and Multi-Agent Systems (AAMAS2020) in New Zealand. Agent technology and Intelligent Agents in Healthcare have gained well established applications to solve various problems in the healthcare domain. This topical collection presents seven articles that discuss different applications of agent systems, in diverse medical areas including circadian rhythm modelling, patient trajectories, emotional expression, brain MRI segmentation, health decision support system, healthcare cognitive services, and healthcare digital twins.

A wide range of submissions were received for the HIMAS2020 workshop. The call for papers attracted leading research in healthcare multi-agent-systems. The peer review process with rigorous feedback and scoring reduced the workshop acceptance rate, leading to a selected number of best submissions which were accepted for workshop participation and presentation. Furthermore for this accompanying topical

This article is part of the Topical Collection on HIMAS 2020

Neil Vaughan

N.Vaughan@exeter.ac.uk

1 Royal Academy of Engineering Research Fellow, University of Exeter, Exeter, England, UK

2 Eurecat, eHealth Unit, Centre Tecnològic de Catalunya, Barcelona, Spain

3 University of Modena and Reggio Emilia, Modena, Italy

4 University of Bologna, Bologna, Italy

5 University of Applied Sciences Western Switzerland, Sierre, Switzerland collection publication of proceedings, these seven papers were selected.

All the papers included for publication in this issue demonstrate the importance and vital contribution of multi agent systems to healthcare. The papers also outline various open problems and research questions that must continue to be addressed in our research community as future or ongoing research works.

This year's HIMAS2020 workshop was held entirely online, as opposed to the original venue in New Zealand, an unconventional approach due to the severe affects and travel restriction caused by the world outbreak of SARS-CoV-2 ("novel Coronavirus").

This introductory article to the topical collection also serves to celebrate the success and track record of this workshop series. This International Workshop on Healthcare Intelligent Multi-Agent Systems (HIMAS2020) extends the long track record and experience in our organising team with the regular string of related workshop series dating back to 2002. The first workshops on agents applied in health care (A2HC) were held at ECAI 2002 in Lyon, France. Then followed ECAI 2004 in Valencia, Spain, IJCAI 2005 in Edinburgh, Scotland, ECAI 2006 in Riva del Garda, Italy, AAMAS 2008 in Estoril, Portugal, and eHealth 2010 in Casablanca, Morocco. Then the workshop became Agents for Ambient Assisted Living (AAAL11 and 12) held with PAAMS 2011 and 2012, AAMAS 2012 in Valencia, Spain, and AIME 2013 in Murcia, Spain. Agents and Multi-Agent Systems for Ambient Assisted Living and eHEALTH 2013. The Multi-Agent Systems for Healthcare (MASH'14) (held with AAMAS 2014). A2HC 2015 in Istanbul, Turkey, and A2HC 2017 in São Paulo, Brazil. Also, the Joint Workshop on Artificial Intelligence for Health (AIH 2018) at IJCAI. Finally, our workshop that these proceedings accompany: HIMAS2020, part of A A M A S 2020 , New Zealand. It is the organisers ongoing intention that each workshop built upon the last, strengthening foundations for future 
workshops to be announced extending this research community further.

Finally, we express gratitude to the many scientists who contributed in some way towards delivering HIMAS2020: presenters, reviewers, organisers past, present and future, schedulers, publishers, editors,
HIMAS chairs, AAMAS chairs, research readers and authors.

Publisher's Note Springer Nature remains neutral with regard to jurisdictional claims in published maps and institutional affiliations. 